\title{
Alpha-smooth Muscle Actin Expression in the Stroma Predicts Resistance to Trastuzumab in Patients with Early-stage HER2-positive Breast Cancer
}

Ioannis A. Vathiotis,2, Myrto K. Moutafi, ${ }^{1,2}$, Prajan Divakar ${ }^{3}$, Thazin Nwe Aung ${ }^{1,2}$, Tao Qing ${ }^{2,4}$, Aileen Fernandez ${ }^{1,2}$, Vesal Yaghoobi, ${ }^{1,2}$, Sarra El-Abed ${ }^{5}$, Yingbo Wang ${ }^{6}$, Sebastien Guillaume ${ }^{7}$, Paolo Nuciforo ${ }^{8}$, Jens Huober ${ }^{9}$, Serena Di Cosimo ${ }^{10}$, Sung-Bae Kim ${ }^{11}$, Nadia Harbeck ${ }^{12}$, Henry Gomez ${ }^{13}$, Saba Shafi ${ }^{1,2}$, Konstantinos N. Syrigos ${ }^{14}$, George Fountzilas ${ }^{15,16}$, Christos Sotiriou ${ }^{7}$, Lajos Pusztai ${ }^{2,4}$, Sarah Warren ${ }^{3}$, and David L. Rimm ${ }^{1,2}$

\section{ABSTRACT}

Purpose: The companion diagnostic test for trastuzumab has not changed much in the last 25 years. We used high-plex digital spatial profiling to identify biomarkers besides HER2 that can help predict response to trastuzumab in HER2-positive breast cancer.

Experimental Design: Fifty-eight protein targets were measured in three different molecularly defined compartments by the NanoString GeoMx Digital Spatial Profiler (DSP) in a tissue microarray containing 151 patients with breast cancer that received adjuvant trastuzumab as part of the Hellenic Cooperative Oncology Group 10/05 clinical trial. Promising candidate biomarkers were orthogonally validated with quantitative immunofluorescence (QIF). RNA-sequencing data from the Neoadjuvant Lapatinib and/or Trastuzumab Treatment Optimisation Study (NeoALTTO) were accessed to provide independent cohort validation. Disease-free survival (DFS) was the main outcome assessed. Statistical analyses were performed using a

\section{Introduction}

Approximately $15 \%$ to $20 \%$ of women diagnosed with breast cancer have HER2-positive disease, defined as evidence of HER2

'Department of Pathology, Yale School of Medicine, New Haven, Connecticut. ${ }^{2}$ Yale Cancer Center, Yale School of Medicine, New Haven, Connecticut. ${ }^{3}$ NanoString Technologies, Seattle, Washington. ${ }^{4}$ Section of Medical Oncology, Department of Internal Medicine, Yale School of Medicine, New Haven, Connecticut. ${ }^{5}$ Breast International Group, Brussels, Belgium. ${ }^{6}$ Novartis Pharma AG, Basel, Switzerland. ${ }^{7}$ Institut Jules Bordet, Université Libre de Bruxelles (ULB), Brussels, Belgium. ${ }^{8}$ Molecular Oncology Group, Vall d'Hebron Institute of Oncology (VHIO), Barcelona, Spain. ${ }^{9}$ Department of Obstetrics and Gynaecology of the University of Ulm, Ulm, Germany. ${ }^{10}$ Fondazione IRCCS Instituto Nazionale dei Tumori, Milano, Italy. "Asan Medical Center, University of Ulsan College of Medicine, Seoul, Republic of South Korea. ${ }^{12}$ Breast Center, Ludwig-Maximilians-University, University Hospital, Munich, Germany. ${ }^{13}$ Instituto Nacional de Enfermedades Neoplasicas, Lima, Peru. ${ }^{14}$ Department of Medicine, National and Kapodistrian University of Athens School of Medicine, Athens, Greece. ${ }^{15}$ Aristotle University of Thessaloniki, Thessaloniki, Greece. ${ }^{16}$ German Oncology Center, Limassol, Cyprus.

Note: Supplementary data for this article are available at Clinical Cancer Research Online (http://clincancerres.aacrjournals.org/).

Corresponding Author: David L. Rimm, Department of Pathology, Yale School of Medicine, P.O. Box 208023, New Haven, CT 06520-8023. Phone: 203-737 4204; E-mail: david.rimm@yale.edu

Clin Cancer Res 2021;27:6156-63

doi: $10.1158 / 1078-0432 . C C R-21-2103$

(C)2021 American Association for Cancer Research two-sided test $(\alpha=0.05)$ and multiple testing correction (Benjamini-Hochberg method, FDR $<0.1$ ).

Results: By DSP, high expression of alpha-smooth muscle actin $(\alpha$-SMA), both in the leukocyte and stromal compartments, was associated with shorter DFS in univariate analysis $(P=0.002$ and $P=0.023$, respectively). High $\alpha$-SMA expression in the stroma was validated by QIF after controlling for estrogen receptor and progesterone receptor status [HR, 3.12; 95\% confidence interval (CI), $1.12-8.68 ; P=0.029]$ showing recurrence on trastuzumab in the same cohort. In the NeoALTTO cohort, elevated levels of ACTA2 were predictive for shorter DFS in the multivariate analysis (HR, 3.21; 95\% CI, 1.14-9.05; $P=0.027$ ).

Conclusions: This work identifies $\alpha$-SMA as a novel, easyto-implement biomarker of resistance to trastuzumab that may be valuable in settings where trastuzumab is combined with other therapies. overexpression measured by IHC (3+) or by FISH (HER2 copy number $\geq 6$ or HER2/CEP17 ratio $\geq 2.0$ ); HER2 positivity has been associated with aggressive biological behavior and worse clinical outcomes (1-6). Trastuzumab (herceptin) is a humanized IgG1 kappa $\mathrm{mAb}$ that binds to the extracellular domain of the HER2 receptor. It interferes with HER2 signaling via several mechanisms, including inhibition of receptor dimerization, prevention of extracellular domain shedding, endocytotic destruction of the receptor and antibody-dependent cell-mediated cytotoxicity (ADCC; ref. 7). Since its first FDA approval in 1998, trastuzumab, in conjunction with chemotherapy, has been the standard of care in HER2positive breast cancer (8-13).

Prescription of trastuzumab has required a companion diagnostic test to select patients that would benefit. The current assay combination of IHC, then FISH has high sensitivity (as high as 95\%) but relatively low specificity and is used in effort to leave no patient behind, although many patients may not benefit from the drug $(1,2)$. Still, 26\% to $31 \%$ of patients with early-stage HER2-positive tumors relapse within 10 years from surgery, underscoring the need to identify novel biomarkers to stratify the risk of disease recurrence $(14,15)$. In addition, in the presence of many other drugs that address this target, it would be valuable to augment the testing regimen to find patients likely to be resistant to trastuzumab.

Here, we used the GeoMx Digital Spatial Profiler (DSP; NanoString Technologies) as a discovery tool to find biomarkers associated with resistance to trastuzumab, or recurrence, in patients with early-stage HER2-positive breast cancer. Then, we used mRNA data from the 


\section{Translational Relevance}

Since 1998, trastuzumab has been the standard of care for patients with HER2-positive breast cancer. We used high-plex digital spatial profiling to identify biomarkers besides HER2 independently associated with response to trastuzumab in patients with early-stage HER2-positive breast cancer. High expression of alpha-smooth muscle actin ( $\alpha$-SMA) within the stromal compartment was significantly associated with shorter disease-free survival. This finding was validated by quantitative immunofluorescence in the same cohort. Furthermore, RNAsequencing data from the Neoadjuvant Lapatinib and/or Trastuzumab Treatment Optimisation Study clinical trial indicated that increased expression of ACTA2 mRNA was predictive for shorter disease-free survival. This work highlights the role of cancer-associated fibroblasts that reside within the tumor microenvironment (TME) in modeling response to trastuzumab. It also identifies $\alpha$-SMA as a novel biomarker of resistance to trastuzumab, with the potential to evolve into an easy-to-implement, TME-based companion diagnostic test.

Neoadjuvant Lapatinib and/or Trastuzumab Treatment Optimisation Study (NeoALTTO) to validate our findings.

\section{Materials and Methods \\ Patient cohorts and samples}

Protein expression was analyzed in prospectively collected, pretreatment fresh-frozen paraffin-embedded tumor specimens, represented in a tissue microarray (TMA; YTMA247), from 180 patients with early-stage HER2-positive breast cancer treated with trastuzumab in the adjuvant setting between 2005 and 2008, as part of the Hellenic Cooperative Oncology Group (HeCOG) 10/05 phase III clinical trial (16). For TMA construction, hematoxylin and eosin-stained slides were reviewed by a board-certified pathologist to select representative tumor areas. Then, 0.6-mm-diameter tumor cores were extracted from each block and arrayed in four recipient master blocks (17). Core selection was not based on specific tumor segments or location. For all experiments two slides derived from different blocks of YTMA247, each containing one nonadjacent tumor core per patient, were assessed. A total of 151 patients had adequate histospots for protein quantification (Table 1). HeCOG 10/05 study protocol was approved by the Institutional Review Boards of all participating centers. Written informed consent or waiver of consent was provided by all the patients.

A validation mRNA-sequencing dataset of 254 patients was obtained from the NeoALTTO clinical trial (18). This trial enrolled 455 patients with early-stage HER2-positive breast cancer. All patients were randomized between 2008 and 2010 to neoadjuvant lapatinib, trastuzumab, or their combination; after surgery, patients received adjuvant chemotherapy followed by the same targeted therapy as in the neoadjuvant phase to 52 weeks. RNA-sequencing (RNA-seq) data from baseline biopsies and matching clinical data were available for 254 patients (Table 1). Additional details on sample processing and sequencing can be found elsewhere (19).

The study was approved by the Yale Human Investigation Committee protocol No. 9505008219 and conducted in accordance with the Declaration of Helsinki.
Table 1. Clinicopathologic characteristics of the discovery (YTMA247) and validation cohorts (NeoALTTO).

\begin{tabular}{|c|c|c|}
\hline Characteristic & $\begin{array}{l}\text { Discovery cohort } \\
\text { (HeCOG 10/05), } \\
N(\%)\end{array}$ & $\begin{array}{l}\text { Validation cohort } \\
\text { (NeoALTTO), } \\
N(\%)\end{array}$ \\
\hline \multicolumn{3}{|l|}{ Age } \\
\hline Median (range) & $54(25-79)$ & $49(23-79)$ \\
\hline \multicolumn{3}{|l|}{ Stage } \\
\hline 1 & $18(11.9)$ & $0(0)$ \\
\hline ॥ & $71(47.0)$ & $151(59.4)$ \\
\hline III & $60(39.7)$ & $103(40.6)$ \\
\hline \multicolumn{3}{|l|}{ Grade } \\
\hline 1 & $0(0)$ & $6(2.4)$ \\
\hline 2 & $64(42.4)$ & $97(38.2)$ \\
\hline 3 & $87(57.6)$ & $122(48.0)$ \\
\hline \multicolumn{3}{|l|}{ Estrogen receptor } \\
\hline Positive & $88(58.3)$ & $126(49.6)$ \\
\hline Negative & $63(41.7)$ & $128(50.4)$ \\
\hline \multicolumn{3}{|c|}{ Progesterone receptor } \\
\hline Positive & $77(51.0)$ & $97(38.2)$ \\
\hline Negative & $74(49.0)$ & $157(61.8)$ \\
\hline \multicolumn{3}{|l|}{ HER2 } \\
\hline Positive & $151(100)$ & $254(100)$ \\
\hline Negative & $0(0)$ & $0(0)$ \\
\hline \multicolumn{3}{|l|}{ Arm } \\
\hline Trastuzumab & $151(100)$ & $79(31.1)$ \\
\hline Lapatinib & $0(0)$ & $89(35.0)$ \\
\hline Combination & $0(0)$ & $86(33.9)$ \\
\hline \multicolumn{3}{|l|}{ Disease recurrence } \\
\hline Yes & $19(12.6)$ & $53(20.9)$ \\
\hline No & $132(87.2)$ & 201 (79.1) \\
\hline
\end{tabular}

\section{Digital spatial profiling}

YTMA247 slides were deparaffinized, subjected to antigen retrieval and incubated overnight with three fluorescent-labeled visualization antibodies to detect epithelial tumor cells (pan-cytokeratin; PanCK), tumor-infiltrating leukocytes (CD45) and tumor-infiltrating macrophages (CD68), along with a cocktail of 58 unique, previously validated, oligonucleotide-labeled antibodies (Supplementary Table S1). Nuclear staining was performed, and tissue was fixed. Next, slides were loaded in the GeoMx DSP instrument and scanned to produce digital fluorescent images of the tissue. Each TMA spot was represented by a unique region of interest (ROI). Each ROI was segmented in three molecularly defined compartments based on fluorescent colocalization, including a tumor compartment $(\mathrm{PanCK}+)$, a leukocyte compartment $\left(\mathrm{CD} 5^{+} / \mathrm{CD} 68^{-}\right)$and a macrophage compartment $\left(\mathrm{CD}^{+} 8^{+}\right)$. These molecular compartments are called areas of interest (AOI; Fig. 1). Oligos from each AOI were released upon exposure to UV light, by sequential assignment of the tumor, leukocyte, and macrophage compartments. Photocleaved oligos were collected via microcapillary tube inspiration and transferred into a 96-well plate. Oligos were then hybridized to four-color, six-spot optical barcodes and finally counted in the nCounter System (NanoString Technologies). Digital counts were calibrated for optical barcode binding efficiency, quality controlled and normalized to internal spike-in controls. To account for stromal protein expression, leukocyte and macrophage compartments were analyzed in aggregate. To evaluate assay performance, we studied the signal to background ratio for each target per AOI, by dividing the spike-in normalized target count by its respective background level. Background levels were established by calculating the geometric mean of nonspecific counts from three 

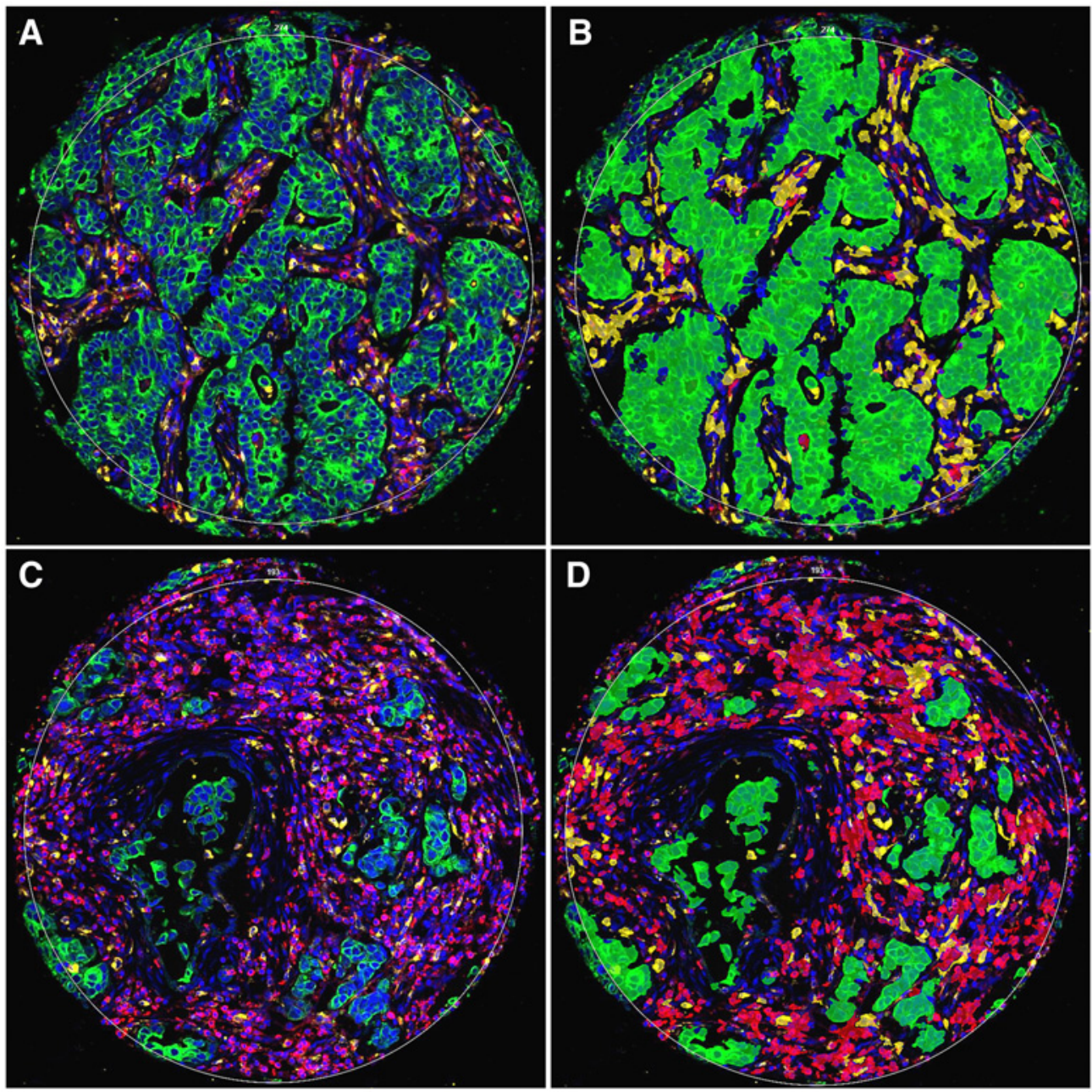

Figure 1.

Tissue segmentation in the GeoMx DSP instrument. Each TMA spot represented a unique ROI. In each ROI, proteins were quantified in three different enriched compartments or areas of interest [AOl; tumor ( $\mathrm{PanCK}+$ ) in green, leukocyte $\left(\mathrm{CD} 45^{+} / \mathrm{CD}^{-} 8^{-}\right)$in red and macrophage $\left(\mathrm{CD} 68^{+}\right)$in yellow]. To assess stromal protein expression, leukocyte and macrophage AOls were analyzed in aggregate. Representative ROIs of YTMA247: fluorescent images $\mathbf{A}$ and $\mathbf{C}$ and derived compartmentalized images $\mathbf{B}$ and $\mathbf{D}$.

negative isotype controls (Mouse IgG1, Mouse IgG2a, and Rabbit IgG) in each AOI. All antibodies exhibited acceptable dynamic range with most of them showing high signal relative to background across different AOIs (Supplementary Fig. S1). To ensure assay reproducibility we compared normalized counts obtained from nonadjacent tumor cores stained in different blocks of YTMA247, on different days. As shown in Supplementary Fig. S2, individual targets (e.g., HER2, Ki67) were highly concordant between runs $(R>0.80)$.

\section{Multiplexed quantitative immunofluorescence}

A multiplexed immunofluorescence staining protocol for simultaneous detection of PanCK, CD45, and $\alpha$-SMA was performed in serial sections of YTMA247. Fluorescence images were acquired using a PM2000 system (Navigate Biopharma) and signal quantification for $\alpha$-SMA was determined by the automated quantitative analysis (AQUA) method of QIF, as described previously $(20,21)$. Target was measured within two different compartments: the tumor mask, created by binarizing and dilating the cytokeratin signal and the stromal mask, created by excluding the tumor mask from DAPI mask, which was created by binarizing and dilating the DAPI signal, that represented the total tissue. QIF scores were generated by dividing the summed pixel intensities for the marker of interest by the area of the compartment in which it was measured and then normalized to the exposure time and bit depth at which the images were captured. Cases with staining artifacts or less than $2 \%$ compartment area were systematically excluded after visual inspection.

\section{RNA-seq data analysis}

The paired-end fastq files of NeoALTTO RNA-seq data were available at Gene Expression Omnibus repository (accession number GSE116335; ref. 22). Sequencing adapters and low-quality reads were trimmed with Trimmomatic (v0.36; ref. 23). The remained reads of 254 baseline samples were mapped to human reference genome (vGRCh38) using STAR (v2.7.1; ref. 24). Raw sequencing counts were obtained using RSEM (v1.3.0), normalized to reads per million and further transformed to $\log _{2}$ scale (25). 


\section{Statistical analysis}

Normalized digital counts obtained by different blocks of YTMA247 were averaged. Pearson correlation coefficient was used to analyze the linear association between two continuous variables. Each case was stratified into high or low expression group using two exploratory cut-off points, median and top tertile. Disease-free survival (DFS) analysis was performed using a univariate Cox regression model. Multiplicity adjustments for DFS associations were applied using the Benjamini-Hochberg method (FDR $<0.1$ ), considering the number of tests performed per compartment (tumor, CD45, CD68, and stroma), and separately for median and top tertile cut-off points.

QIF scores of different blocks of YTMA247 were also averaged. Patients were separated into two groups according to whether the expression of $\alpha$-SMA protein within the stromal compartment was higher ( $\alpha$-SMA high) or not higher ( $\alpha$-SMA low) than its median expression. DFS between the two groups was compared using both a univariate and a multivariate Cox regression model, after adjusting for estrogen receptor (ER) and progesterone receptor (PR) status.

In the NeoALTTO cohort, patients were separated into two groups according to whether the expression of ACTA2 gene was higher (ACTA2 high) or not higher (ACTA2 low) than its median expression within each treatment arm. DFS between the two groups was compared by a Cox regression model, after adjusting for ER and PR status. To assess the predictive value of ACTA2 an interaction test was performed using the lapatinib arm as the placebo. All hypothesis testing was performed at a two-sided significance level of $\alpha=0.05$. Statistical analysis was performed using R 3.6.3.

\section{Results}

After removing positive, negative controls and cytokeratin, we assessed 51 individual protein targets in four compartments, resulting in 204 candidate biomarkers per patient. Unsupervised hierarchical clustering revealed four predominant marker clusters; $\alpha$-SMA clustered together with CD44 and CD45 in nontumor compartments, representing the most highly overexpressed targets in this dataset (Supplementary Fig. S3). Using two exploratory cut-off points, we found 20 markers associated with DFS in spatial context by unadjusted univariate analysis (Table 2). After adjustment for multiple testing, two markers remained significantly negatively associated with DFS, $\alpha$-SMA, and CTL-associated protein 4 (CTLA-4), both measured in the leukocyte compartment $(P=0.045$ and $P=0.084$, respectively). As $\alpha$-SMA is a marker for cancer-associated fibroblasts (CAF), measurements in the leukocyte compartment captured part of stromal protein expression, rather than reflecting true colocalization with immune cells. Indeed, digital counts of $\alpha$-SMA in the leukocyte compartment were correlated with digital counts in the macrophage $(R=0.70$, $P=2.1 \mathrm{e}-15)$ and stromal compartments $(R=0.88, P<2.2 \mathrm{e}-16$; Supplementary Fig. S4A and S4B). Accordingly, increased $\alpha$-SMA expression within the stromal compartment was associated with significantly shorter DFS in unadjusted univariate analysis (median cut-off point; $P=0.023$ ). Digital counts of $\alpha$-SMA in the stroma showed positive correlation with those of CTLA-4 in the leukocyte compartment $(R=0.73, P<2.2 \mathrm{e}-16)$ but no correlation with leukocytic infiltration, as reflected by digital counts of CD45 in the leukocyte compartment $(R=0.03, P=0.739$; Supplementary Fig. S4C and S4D).

To validate the association of $\alpha$-SMA in the stroma with DFS, we assessed its expression using an orthogonal, fluorescent-based method in the same cohort (Fig. 2A-C). We detected a range of expression of this marker across the cohort, with most cases showing stromal
Table 2. Markers significantly associated with DFS under treatment with trastuzumab in univariate analysis.

\begin{tabular}{|c|c|c|c|c|}
\hline Compartment & Marker & Cutoff & $\begin{array}{l}\text { Log-rank } \\
P\end{array}$ & $\begin{array}{l}\text { Univariate HR } \\
(95 \% \mathrm{Cl})\end{array}$ \\
\hline \multirow[t]{2}{*}{ Tumor } & ID01 & Top tertile ${ }^{a}$ & 0.011 & $0.12(0.02-0.87)$ \\
\hline & FAP-alpha & Median & 0.026 & $0.30(0.10-0.93)$ \\
\hline \multirow[t]{5}{*}{ Leukocyte } & CTLA4 & Top tertile ${ }^{a}$ & 0.002 & $6.41(1.70-24.22)$ \\
\hline & SMA & Top tertile & 0.002 & $6.37(1.68-24.07)$ \\
\hline & ER-alpha & Top tertile & 0.021 & 3.83 (1.12-13.09) \\
\hline & ICOS & Median & 0.024 & $0.20(0.04-0.95)$ \\
\hline & IDO1 & Median & 0.029 & $0.21(0.05-0.99)$ \\
\hline \multirow[t]{8}{*}{ Macrophage } & GZMB & Median $^{a}$ & 0.005 & $0.15(0.03-0.69)$ \\
\hline & PD-L2 & Top tertile & 0.008 & 3.91 (1.31-11.67) \\
\hline & ER-alpha & Median & 0.021 & $4.02(1.12-14.43)$ \\
\hline & ICOS & Median & 0.022 & $0.25(0.07-0.90)$ \\
\hline & PD-L1 & Top tertile & 0.027 & 0.14 (0.02-1.07) \\
\hline & B2M & Median & 0.028 & $0.26(0.07-0.65)$ \\
\hline & IDO1 & Top tertile & 0.033 & $0.15(0.02-1.13)$ \\
\hline & FOXP3 & Top tertile & 0.033 & $2.99(1.04-8.63)$ \\
\hline \multirow[t]{5}{*}{ Stroma } & SMA & Median & 0.023 & $4.94(1.07-22.86)$ \\
\hline & ICOS & Median & 0.024 & $0.20(0.04-0.94)$ \\
\hline & PD-L1 & Median & 0.026 & $0.21(0.04-0.96)$ \\
\hline & IDO1 & Median & 0.029 & $0.21(0.05-0.98)$ \\
\hline & VISTA & Median & 0.031 & $0.22(0.05-1.00)$ \\
\hline
\end{tabular}

${ }^{a}$ The marker was significant using both cutoff points, median and top tertile.

localization for $\alpha$-SMA (Fig. 2D). We observed a strong agreement between digital counts and QIF scores of $\alpha$-SMA within the stromal compartment $(R=0.68, P=3.3 \mathrm{e}-14)$, featuring the high concordance between the two assays (Fig. 2E). Then, using the median cut-off point, we confirmed that patients with high stromal $\alpha$-SMA expression performed significantly worse in terms of DFS as compared with patients with low stromal expression of $\alpha$-SMA [HR, 3.12; 95\% confidence intervals (CIs), $1.12-8.68 ; P=0.029$ ] by univariate analysis (Fig. 2F). In the multivariate analysis, including two clinical prognostic factors (ER and PR status), high expression of $\alpha$-SMA in the stroma was the only factor that showed significant association with shorter DFS (HR, 2.91; 95\% CI, 1.04-8.17; $P=0.042$ ).

Finally, to validate the predictive relevance of $\alpha$-SMA overexpression in patients with HER2-positive breast cancer treated with trastuzumab, we analyzed RNA-seq data from the NeoALTTO clinical trial. Once again, using the median cut-off point, we found that elevated levels of ACTA2 mRNA were associated with shorter DFS (HR, 3.21; 95\% CI, 1.14-9.05; $P=0.027$ ) in patients that received neoadjuvant trastuzumab by multivariate analysis, after controlling for ER and PR status (Fig. 3A). Notably, this was not the case for patients either in the lapatinib (HR, 0.77; 95\% CI, 0.33-1.79; $P=0.543$, Fig. 3B) or in the combination arm (HR, 0.65; 95\% CI, 0.21-2.04; $P=0.469$; Fig. 3C). After performing an interaction test, we found that increased ACTA2 expression was predictive for disease recurrence in patients with early-stage HER2-positive breast cancer treated with trastuzumab $(P=0.049)$

\section{Discussion}

In this study, we used the DSP technology to discover novel biomarkers of response, or resistance to trastuzumab in a clinical trial cohort of 151 patients with HER2-positive breast cancer. By combining high-plex capacity with spatial resolution, we found 20 candidate biomarkers that were associated with DFS in univariate analysis. 

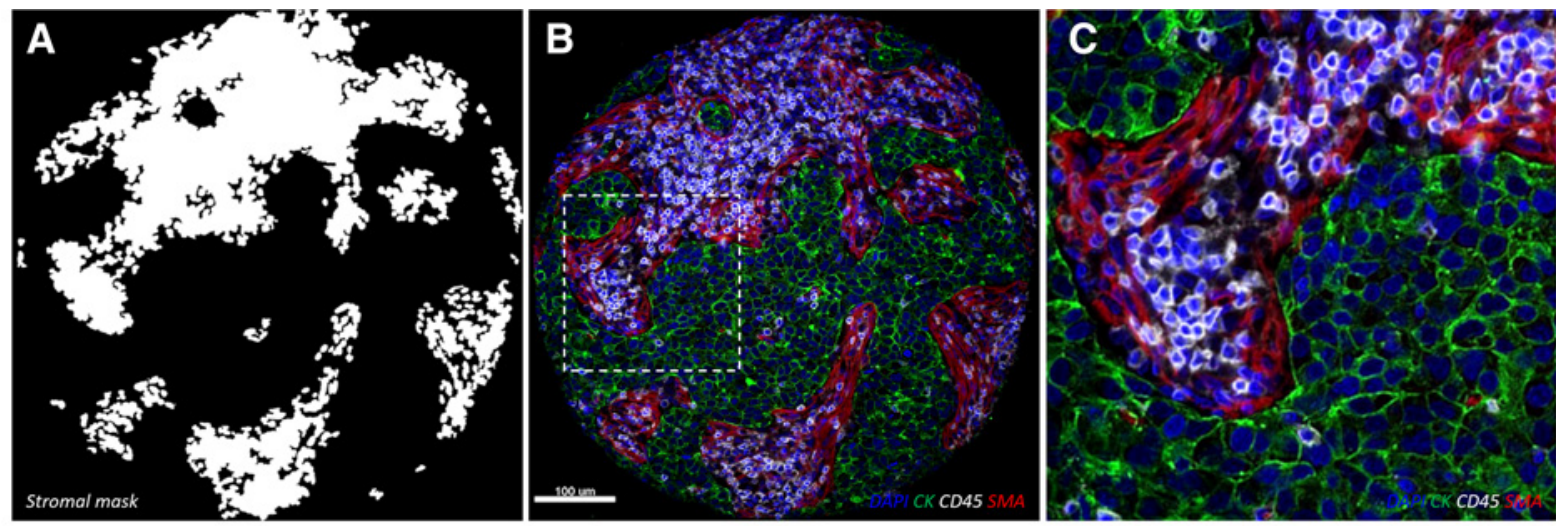

D

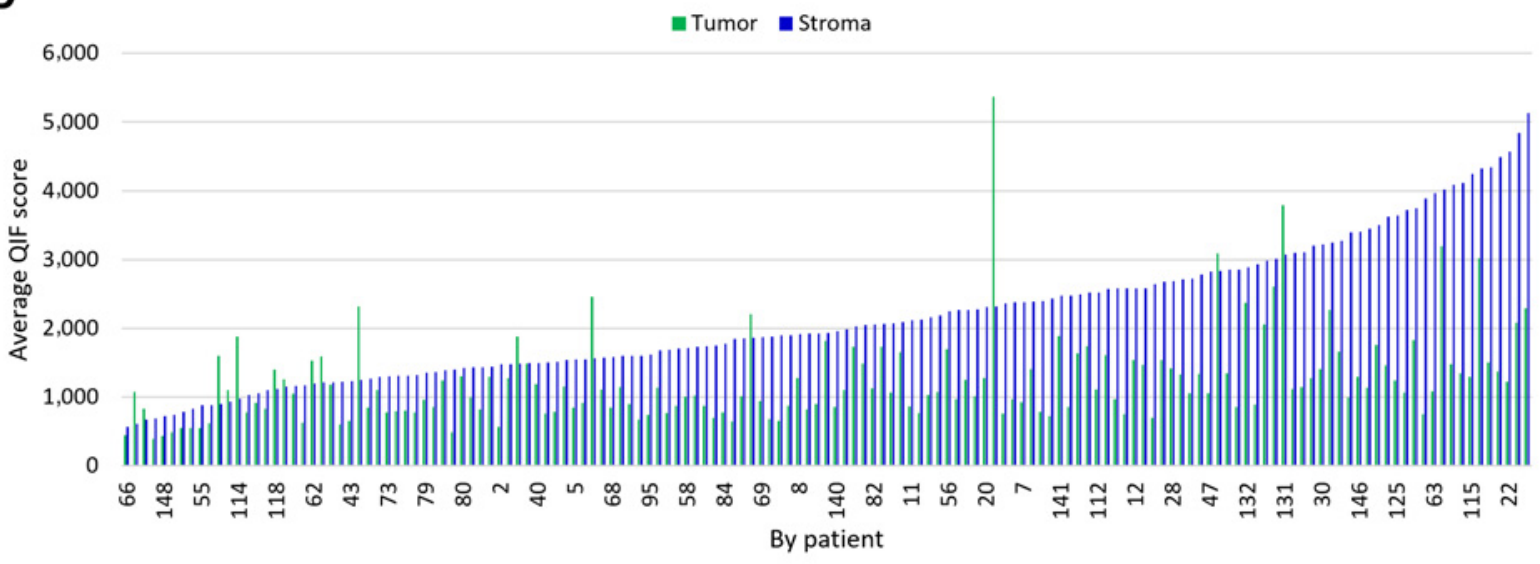

\section{E}

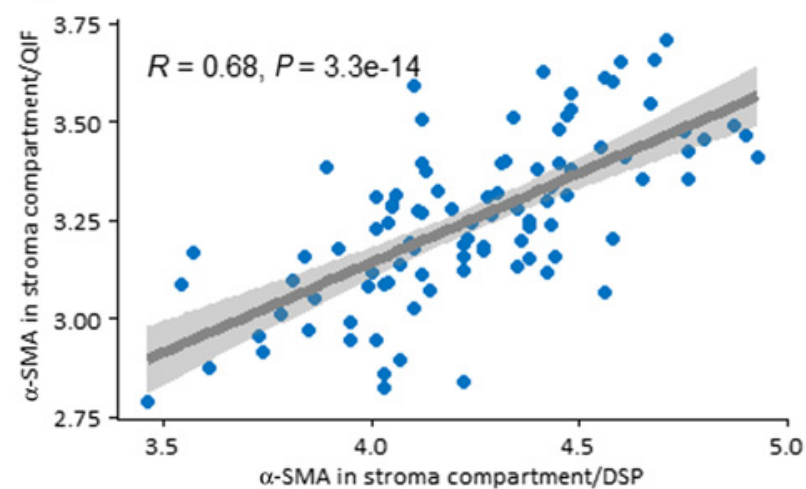

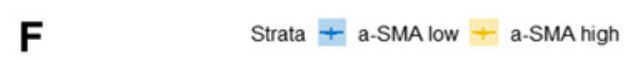
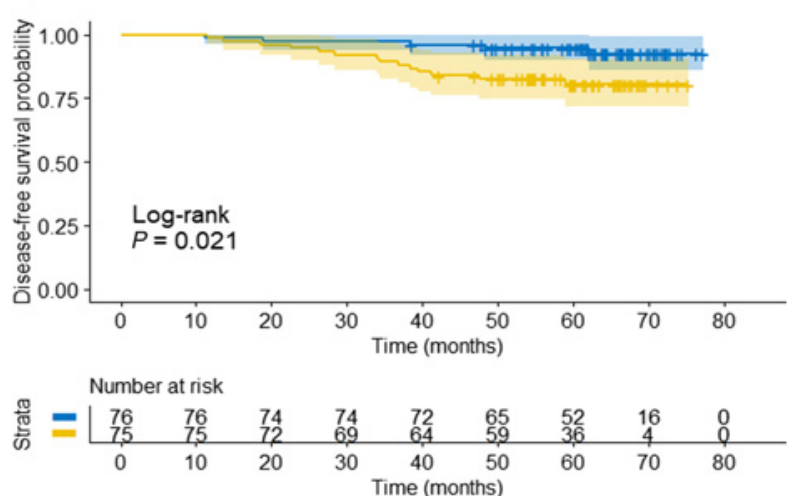

Figure 2.

Orthogonal validation of $\alpha$-SMA in stroma by the AQUA method of QIF. Representative images showing the stromal compartment (A) and $\alpha$-SMA staining pattern (B and C). D, QIF scores of $\alpha$-SMA within the tumor and stromal compartment. E, Scatter plot demonstrating the correlation between digital counts of $\alpha$-SMA in stroma by DSP and QIF. F, Kaplan-Meier plot for DFS with respect to $\alpha$-SMA expression in the stroma.

Perhaps, the most compelling finding was the identification of high levels of $\alpha$-SMA within the stromal compartment as a predictor of unfavorable clinical outcome, in terms of shorter DFS, under treatment with adjuvant trastuzumab. We confirmed its association with DFS with an orthogonal method and after controlling for clinical prognostic factors. Furthermore, using RNA-seq data from the NeoALTTO clinical trial, we validated the predictive value of this biomarker in an independent clinical trial cohort and expanded its applicability to accommodate patients with breast cancer receiving trastuzumab in the neoadjuvant setting. Taken together, these results amplify previous findings showing that CAFs within the tumor microenvironment (TME) are important mediators of resistance to trastuzumab (26-28).

We believe this is particularly interesting because $\alpha$-SMA, as a surrogate marker for CAFs, is easily assessed by routine IHC testing. CAFs represent the most abundant cell type of the breast TME and 
A

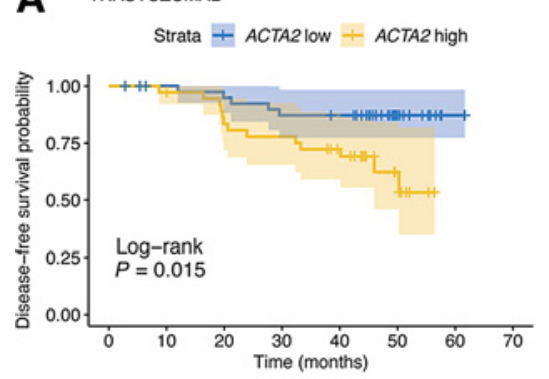

B
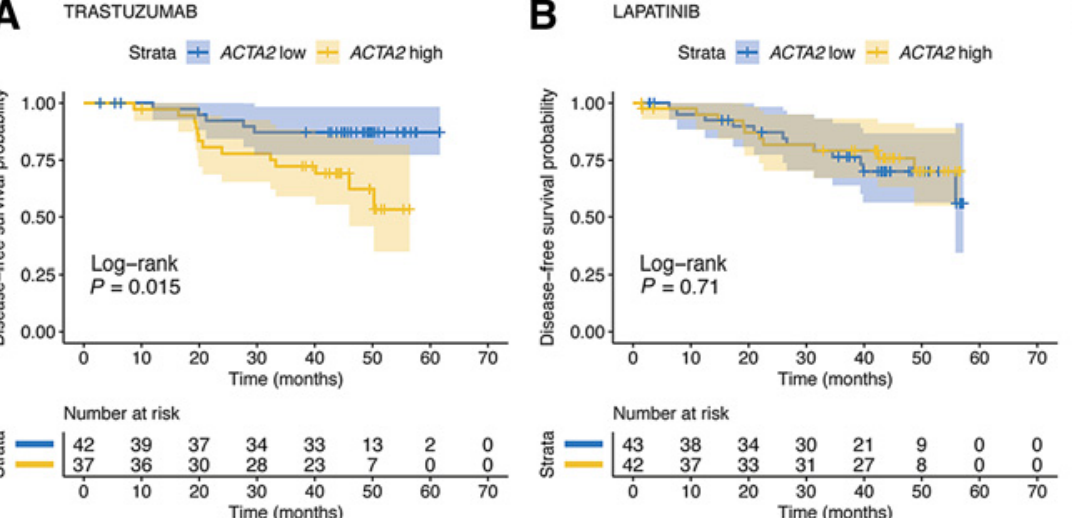
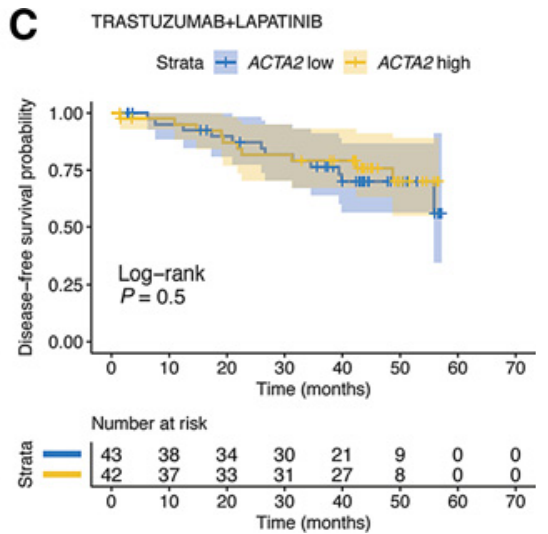

Figure 3.

Kaplan-Meier curves for DFS between patients with high and low ACTA2 expression in NeoALTTO cohort. Trastuzumab arm (A); lapatinib arm (B); trastuzumab + lapatinib arm (C).

their composition appears to change during the course of the disease, with implications to outcome (29). A recent study in breast cancer revealed at least three spatially and functionally distinct subsets of CAFs by single-cell RNA-seq, however expression patterns of ACTA2 were broad and not characteristic of a single CAF subpopulation (30). On the basis of the levels of expression of six surface markers, Costa and colleagues identified four CAF subsets that accumulated differentially in breast cancer subtypes and normal juxta-tumors; HER2positive breast cancer was enriched in CAF-S4 subset, which stained positive for $\alpha$-SMA (31). The assessment of a-SMA is already done in nearly all Clinical Laboratory Improvement Amendments IHC labs because this marker is used as an aid for diagnosis of some tumor types. After future development and validation of this biomarker, it is easy to envision broad adoption for selection of patients that are HER2 positive but need more than just trastuzumab. These patients may benefit from more intensive therapeutic approaches, including dual HER2 inhibition or treatment with an alternative anti-HER2 agent. Building on the immunosuppressive role of CAFs, such patients may be candidates for future clinical trials involving immune checkpoint inhibition, or novel approaches that will target CAFs in particular.

CAFs have been shown to promote resistance to trastuzumab in several preclinical breast cancer models $(32,33)$. Epitope masking could potentially explain CAF-mediated resistance to trastuzumab $(34,35)$. Adverse remodeling of the extracellular matrix by CAFs may limit drug penetration and hinder binding of trastuzumab to the HER2 receptor. This concept is further supported by the fact that the negative effects of increased levels of ACTA2 in patients treated with trastuzumab were alleviated by lapatinib, which is a small-molecule inhibitor targeting the intracellular domain of the receptor $(36,37)$. There is a growing body of evidence that failure to trigger ADCC may also contribute to trastuzumab resistance in patients with increased $\alpha$-SMA in the stroma. CAF secretory functions as well as surface expression of inhibitory immune checkpoints mediate immune reprogramming and induce immunosuppressive changes in the TME (26). Although the expression of $\alpha$-SMA in the stroma did not correlate with leukocytic infiltration, we noted a positive correlation with CTLA- 4 in the leukocyte compartment. Upregulation of CTLA- 4 has been shown to attenuate the immune response and promote peripheral tolerance (38-40). Recently, Fernández-Nogueira and colleagues iden- tified a new, CAF-related escape mechanism for HER2-targeted therapies in breast cancer and suggested that it can be reversed by FGFR inhibition (41).

There are several limitations to this study. First, assessment of $\alpha$-SMA protein in the stroma, by both DSP and AQUA, was done on TMAs. Although we analyzed two nonadjacent tumor cores per patient, we realize that this covers a small percentage of a standard whole tissue section. This is a common initial approach for biomarker studies with the rationale being that if an effect can be seen on TMA, it is likely to be also seen in biopsy specimens in future studies. Furthermore, we have increased confidence in our TMA results because the mRNA-sequencing information was obtained from conventional biopsy whole tissue slides. Second, although we provide indirect evidence for the lack of prognostic value of $\alpha$-SMA by showing no association with outcome in patients treated with either lapatinb or trastuzumab and lapatinib, we have not formally assessed $\alpha$-SMA expression in a cohort of patients with early-stage HER2-positive breast cancer that did not receive adjuvant therapy. We note that to rigorously prove "predictive" value, a "notreatment" arm is required, and it would be challenging or impossible from an ethical perspective to prospectively obtain such information. Another set of limitations is inherent in the current DSP technology. Resolution of the platform is limited to $10 \mu \mathrm{m}$, meaning that some immune cells or other cells within the defined molecular compartments may be misassigned. This limitation is addressed by validation with QIF which has sub-micron resolution, as used in this study. Finally, in this pilot, discovery study, $\alpha$-SMA protein was evaluated only in a single clinical trial cohort of patients with breast cancer. In the future, studies are planned to address the predictive value of this biomarker by IHC in multi-institutional studies and in patients with HER2-positive breast cancer receiving trastuzumab in the metastatic setting, as well as patients receiving trastuzumab for HER2-positive esophageal or gastric cancer.

In conclusion, we identified, characterized, and validated $\alpha$-SMA as a new biomarker, predictive for relapse following treatment with trastuzumab in patients with early-stage HER2-positive breast cancer and laid the groundwork for the generation of a novel TME-based companion diagnostic test. Furthermore, we identified several other promising candidate biomarkers in spatial context that are currently being investigated in parallel to this work. Here, we used the DSP 
platform to discover novel stromal cancer biomarkers. Future studies will focus on the TME, with the aim to unravel the biology behind CAFs and to bring this TME-based companion diagnostic test to the clinic.

\section{Authors' Disclosures}

P. Divakar reports other support from NanoString Technologies, Inc. outside the submitted work. S. El-Abed reports grants from Novartis during the conduct of the study and grants from Pfizer and Roche/Genentech outside the submitted work. Y. Wang reports other support from Novartis during the conduct of the study and other support from Novartis outside the submitted work. S. Guillaume reports othe support from GlaxoSmithKline and Novartis during the conduct of the study and other support from AstraZeneca and Roche outside the submitted work. P. Nuciforo reports grants from Novartis during the conduct of the study and personal fees from Bayer, Merck Sharp \& Dohme, and Targos outside the submitted work. J. Huober reports personal fees and other support from Pfizer and Roche; grants and personal fees from Lilly, Novartis, and Hexal; and personal fees from Gilead, Seagen, AbbVie, AstraZeneca, Merck Sharp \& Dohme, and Eisai; and other support from Daichii outside the submitted work. S. Di Cosimo reports personal fees from Pierre-Fabre and grants from Fondazione AIRC outside the submitted work. S.-B. Kim reports grants from Novartis, Sanofi-Aventis, and Dongkook outside the submitted work; is a consultant in advisory boards for Novartis, AstraZeneca, Lilly, Dae Hwa Pharmaceutical Co. Ltd., ISU Abxis, Daiichi-Sankyo, Beigene, and Pfizer; and holds stocks in Genopeaks and NeogeneTC. N. Harbeck reports personal fees from AstraZeneca, Daiichi-Sankyo, Lilly, Novartis, Roche, Pfizer, Pierre-Fabre, and SeaGen outside the submitted work. G. Fountzilas reports personal fees from Roche during the conduct of the study; personal fees from Pfizer, Novartis, and AstraZeneca outside the submitted work; and stock ownership with Daiichi Sankyo, ARIAD, RFL Holdings, and Formycon. L. Pusztai reports grants and personal fees from Roche Genentech, Pfizer, Bristol Myers Squibb, AstraZeneca, and Merck and grants from Seagen outside the submitted work. S. Warren reports other support from NanoString Technologies during the conduct of the study. D.L. Rimm reports personal fees from NanoString during the conduct of the study; personal fees from AstraZeneca, Cell Signaling Technology, Merck, Monopteros, PAIGE.AI, and Roche; grants from Amgen, Konica Minolta, and Navigate BP; and grants and personal fees from Cepheid and NextCure outside the submitted work. No disclosures were reported by the other authors.

\section{References}

1. Lebeau A, Deimling D, Kaltz C, Sendelhofert A, Iff A, Luthardt B, et al. Her-2/neu analysis in archival tissue samples of human breast cancer: comparison of immunohistochemistry and fluorescence in situ hybridization. J Clin Oncol 2001;19:354-63.

2. Wolff AC, Hammond MEH, Hicks DG, Dowsett M, McShane LM, Allison KH, et al. Recommendations for human epidermal growth factor receptor 2 testing in breast cancer: American society of clinical oncology/college of American pathologists clinical practice guideline update. J Clin Oncol 2013;31:3997-4013.

3. Slamon D, Godolphin W, Jones L, Holt J, Wong S, Keith D, et al. Studies of the HER-2/neu proto-oncogene in human breast and ovarian cancer. Science 1989; 244:707-12.

4. Ross JS, Fletcher JA, Linette GP, Stec J, Clark E, Ayers M, et al. The Her-2/neu gene and protein in breast cancer 2003: biomarker and target of therapy. Oncologist 2003;8:307-25.

5. Schechter AL, Stern DF, Vaidyanathan L, Decker SJ, Drebin JA, Greene MI, et al. The neu oncogene: an erb-B-related gene encoding a $185,000-\mathrm{Mr}$ tumour antigen. Nature 1984;312:513-6.

6. Bargmann CI, Hung MC, Weinberg RA. The neu oncogene encodes an epidermal growth factor receptor-related protein. Nature 1986;319:226-30.

7. Hudis CA. Trastuzumab-mechanism of action and use in clinical practice. N Engl J Med 2007;357:39-51.

8. Slamon DJ, Leyland-Jones B, Shak S, Fuchs H, Paton V, Bajamonde A, et al. Use of chemotherapy plus a monoclonal antibody against HER2 for metastatic breast cancer that overexpresses HER2. N Engl J Med 2001;344:783-92.

9. Piccart-Gebhart MJ, Procter M, Leyland-Jones B, Goldhirsch A, Untch M, Smith I, et al. Trastuzumab after adjuvant chemotherapy in HER2-positive breast cancer. N Engl J Med 2005;353:1659-72.

10. Romond EH, Perez EA, Bryant J, Suman VJ, Geyer CE, Davidson NE, et al. Trastuzumab plus adjuvant chemotherapy for operable HER2-positive breast cancer. N Engl J Med 2005;353:1673-84.

\section{Authors' Contributions}

I.A. Vathiotis: Conceptualization, resources, software, formal analysis, validation, investigation, visualization, methodology, writing-original draft, writing-review and editing. M.K. Moutafi: Investigation, writing-review and editing. P. Divakar: Software, formal analysis, supervision, writing-review and editing. T.N. Aung: Software, formal analysis, writing-review and editing. T. Qing: Software, formal analysis, writing-original draft, writing-review and editing. A. Fernandez: Validation, writing-review and editing. V. Yaghoobi: Validation, writing-review and editing. S. El-Abed: Resources, writing-review and editing. Y. Wang: Resources, writing-review and editing. S. Guillaume: Resources, writing-review and editing P. Nuciforo: Resources, writing-review and editing. J. Huober: Resources, writingreview and editing. S. Di Cosimo: Resources, writing-review and editing. S.-B. Kim: Resources, writing-review and editing. N. Harbeck: Resources, writing-review and editing. H. Gomez: Resources, writing-review and editing. S. Shafi: Validation, writing-review and editing. K.N. Syrigos: Supervision, visualization, writing-review and editing. G. Fountzilas: Resources, visualization, writing-review and editing C. Sotiriou: Resources, supervision, visualization, writing-review and editing L. Pusztai: Resources, formal analysis, supervision, funding acquisition, visualization, project administration, writing-review and editing. S. Warren: Conceptualization, resources, supervision, writing-review and editing. D.L. Rimm: Conceptualization, resources, supervision, funding acquisition, investigation, methodology, project administration, writing-review and editing.

\section{Acknowledgments}

This research was supported by the Breast Cancer Research Foundation (D.L. Rimm and L. Pusztai) and an NCI R01 grant (R01CA219647), to L. Pusztai. I.A. Vathiotis and M.K. Moutafi are supported by a scholarship from the Hellenic Society of Medical Oncologists (HESMO). The authors thank Lori A. Charette and the staff of Yale Pathology tissue services for expert histology services.

The costs of publication of this article were defrayed in part by the payment of page charges. This article must therefore be hereby marked advertisement in accordance with 18 U.S.C. Section 1734 solely to indicate this fact.

Received June 8, 2021; revised July 28, 2021; accepted August 25, 2021; published first August 31, 2021.

11. Slamon D, Eiermann W, Robert N, Pienkowski T, Martin M, Press M, et al. Adjuvan trastuzumab in HER2-positive breast cancer. N Engl J Med 2011;365:1273-83.

12. Gianni L, Eiermann W, Semiglazov V, Lluch A, Tjulandin S, Zambetti M, et al. Neoadjuvant chemotherapy with trastuzumab followed by adjuvant trastuzumab versus neoadjuvant chemotherapy alone, in patients with HER2 positive locally advanced breast cancer (the NOAH trial): a randomised controlled superiority trial with a parallel HER2-negative cohort. Lancet 2010;375:377-84.

13. Wilson FR, Coombes ME, Brezden-Masley C, Yurchenko M, Wylie Q, Douma R, et al. Herceptin ${ }^{\circledR}$ (trastuzumab) in HER2-positive early breast cancer: a systematic review and cumulative network meta-analysis. Syst Rev 2018;7:191.

14. Perez EA, Romond EH, Suman VJ, Jeong J-H, Sledge G, Geyer CE, et al. Trastuzumab plus adjuvant chemotherapy for human epidermal growth factor receptor 2-positive breast cancer: planned joint analysis of overall survival from NSABP B-31 and NCCTG N9831. J Clin Oncol 2014;32:3744-52.

15. Cameron D, Piccart-Gebhart MJ, Gelber RD, Procter M, Goldhirsch A, de Azambuja E, et al. 11 years' follow-up of trastuzumab after adjuvant chemotherapy in HER2-positive early breast cancer: final analysis of the HERceptin Adjuvant (HERA) trial. Lancet 2017;389:1195-205.

16. Fountzilas G, Dafni U, Papadimitriou C, Timotheadou E, Gogas H, Eleftheraki AG, et al. Dose-dense sequential adjuvant chemotherapy followed, as indicated, by trastuzumab for one year in patients with early breast cancer: first report at 5-year median follow-up of a Hellenic Cooperative Oncology Group randomized phase III trial. BMC Cancer 2014;14:515.

17. Camp RL, Charette LA, Rimm DL. Validation of tissue microarray technology in breast carcinoma. Lab Invest 2000;80:1943-9.

18. Baselga J, Bradbury I, Eidtmann H, Di Cosimo S, de Azambuja E, Aura C, et al. Lapatinib with trastuzumab for HER2-positive early breast cancer (NeoALTTO): a randomised, open-label, multicentre, phase 3 trial. Lancet 2012;379:633-40. 
19. Fumagalli D, Venet D, Ignatiadis M, Azim HA, Maetens M, Rothé F, et al. RNA sequencing to predict response to neoadjuvant anti-HER2 therapy: a secondary analysis of the NeoALTTO randomized clinical trial. JAMA Oncol 2017;3:227-34

20. Camp RL, Chung GG, Rimm DL. Automated subcellular localization and quantification of protein expression in tissue microarrays. Nat Med 2002;8 1323-7.

21. MacNeil T, Vathiotis IA, Martinez-Morilla S, Yaghoobi V, Zugazagoitia J, Liu Y, et al. Antibody validation for protein expression on tissue slides: a protocol for immunohistochemistry. BioTechniques 2020;69:460-8.

22. Barrett T, Wilhite SE, Ledoux P, Evangelista C, Kim IF, Tomashevsky M, et al NCBI GEO: archive for functional genomics data sets-update. Nucleic Acids Res 2013;41:D991-5.

23. Bolger AM, Lohse M, Usadel B. Trimmomatic: a flexible trimmer for Illumina sequence data. Bioinformatics 2014;30:2114-20.

24. Dobin A, Davis CA, Schlesinger F, Drenkow J, Zaleski C, Jha S, et al. STAR: ultrafast universal RNA-seq aligner. Bioinformatics 2013;29:15-21.

25. Li B, Dewey CN. RSEM: accurate transcript quantification from RNAseq data with or without a reference genome. BMC Bioinformatics 2011;12:323.

26. Kalluri R. The biology and function of fibroblasts in cancer. Nat Rev Cancer 2016; 16:582-98.

27. Gieniec KA, Butler LM, Worthley DL, Woods SL. Cancer-associated fibroblastsheroes or villains? Br J Cancer 2019;121:293-302.

28. Sahai E, Astsaturov I, Cukierman E, DeNardo DG, Egeblad M, Evans RM, et al. A framework for advancing our understanding of cancer-associated fibroblasts. Nat Rev Cancer 2020;20:174-86.

29. Friedman G, Levi-Galibov O, David E, Bornstein C, Giladi A, Dadiani M, et al Cancer-associated fibroblast compositions change with breast cancer progression linking the ratio of S100A4+ and PDPN+ CAFs to clinical outcome. Nat Cancer 2020;1692-708.

30. Bartoschek M, Oskolkov N, Bocci M, Lövrot J, Larsson C, Sommarin M, et al. Spatially and functionally distinct subclasses of breast cancer-associated fibroblasts revealed by single cell RNA sequencing. Nat Commun 2018;9: 5150 .
31. Costa A, Kieffer Y, Scholer-Dahirel A, Pelon F, Bourachot B, Cardon M, et al. Fibroblast heterogeneity and immunosuppressive environment in human breast cancer. Cancer Cell 2018;33:463-79.

32. Nguyen M, De Ninno A, Mencattini A, Mermet-Meillon F, Fornabaio G, Evans SS, et al. Dissecting effects of anti-cancer drugs and cancer-associated fibroblasts by on-chip reconstitution of immunocompetent tumor microenvironments Cell Rep 2018;25:3884-93.

33. Mao Y, Zhang Y, Qu Q, Zhao M, Lou Y, Liu J, et al. Cancer-associated fibroblasts induce trastuzumab resistance in HER2 positive breast cancer cells. Mol Biosyst 2015;11:1029-40.

34. Pohlmann PR, Mayer IA, Mernaugh R. Resistance to trastuzumab in breast cancer. Clin Cancer Res 2009;15:7479-91.

35. Goutsouliak K, Veeraraghavan J, Sethunath V, De Angelis C, Osborne CK, Rimawi MF, et al. Towards personalized treatment for early stage HER2-positive breast cancer. Nat Rev Clin Oncol 2020;17:233-50.

36. Konecny GE, Pegram MD, Venkatesan N, Finn R, Yang G, Rahmeh M, et al Activity of the dual kinase inhibitor lapatinib (GW572016) against HER-2overexpressing and trastuzumab-treated breast cancer cells. Cancer Res 2006;66: 1630-9.

37. Xia W, Gerard CM, Liu L, Baudson NM, Ory TL, Spector NL. Combining lapatinib (GW572016), a small molecule inhibitor of ErbB1 and ErbB2 tyrosine kinases, with therapeutic anti-ErbB2 antibodies enhances apoptosis of ErbB2 overexpressing breast cancer cells. Oncogene 2005;24:6213-21.

38. Walunas TL, Lenschow DJ, Bakker CY, Linsley PS, Freeman GJ, Green JM, et al CTLA-4 can function as a negative regulator of T cell activation. Immunity 1994 $1: 1405-13$.

39. Chambers CA, Kuhns MS, Egen JG, Allison JP. CTLA-4-mediated inhibition in regulation of $\mathrm{T}$ cell responses: mechanisms and manipulation in tumor immunotherapy. Annu Rev Immunol 2001;19:565-94.

40. Egen JG, Kuhns MS, Allison JP. CTLA-4: new insights into its biological function and use in tumor immunotherapy. Nat Immunol 2002;3:611-8.

41. Fernández-Nogueira P, Mancino M, Fuster G, López-Plana A, Jauregui P, Almendro $\mathrm{V}$, et al. Tumor-associated fibroblasts promote HER2-targeted therapy resistance through FGFR2 activation. Clin Cancer Res 2020;26: 1432-48. 